January, 1997

IFT-P/006/97

solv-int/9701012

\title{
Some comments on the bi(tri)-Hamiltonian structure of Generalized AKNS and DNLS hierarchies'
}

\author{
H.S. Blas Achicf, L.A. Ferreira, J.F. Gomes and A.H. Zimerman \\ Instituto de Física Teórica-UNESP \\ Rua Pamplona 145 \\ 01405-900 São Paulo, Brazil
}

\begin{abstract}
We give the correct prescriptions for the terms involving $\partial_{x}^{-1} \delta(x-y)$, in the Hamiltonian structures of the AKNS and DNLS systems, in order for the Jacobi identities to hold. We also establish that the $\operatorname{sl}(2)$ AKNS and DNLS systems are tri-Hamiltonians and construct two compatible Hamiltonian structures for the $s l(3)$ AKNS system. We also give a derivation of the recursion operator for the $s l(n+1)$ DNLS system.
\end{abstract}

\footnotetext{
${ }^{1}$ Work supported in part by $\mathrm{CNPq}$

${ }^{2}$ Supported by FAPESP
} 
The Hamiltonian structures of the Generalized AKNS and DNLS systems were studied in refs. [1, 2, 3] and [4] respectively. In ref. [2] it was obtained, in a closed form, the recurrence relation for consecutive time evolutions for the Generalized AKNS hierarchy, as well as the first and second Hamiltonian structures. Ref. [3] discussed the compatibility of those two structures. However, some special attention must be paid to the second bracket, since it contains terms involving $\partial_{x}^{-1} \delta(x-y)$. In this letter, we point out that the Jacobi identities for brackets containing such terms are not guaranteed to hold. The quantity $\partial_{x}^{-1} \delta(x-y)$ is not uniquely determined in terms of Heaviside functions, and as shown in (1.12), that undeterminacy involves just one parameter. We show that the $\operatorname{sl}(2)$ AKNS and DNLS systems admit three Hamiltonian structures, and we establish that the the Jacobi identities for such structures are only satisfied for one particular choice of that parameter. Moreover, we also check that those three structures are compatible [5] and so the $s l(2)$ AKNS and DNLS systems are tri-Hamiltonian. In the case of $s l(3)$ AKNS system we have shown that the choice of the above mentioned parameter, in order for the Jacobi identity to hold, is not the same for all terms in the second bracket, involving $\partial_{x}^{-1} \delta(x-y)$. We have determined in fact, that there exists two possible solutions. We also give a derivation of the recursion operator for the $\operatorname{sl}(n+1)$ DNLS system.

\section{AKNS}

The Generalized $\operatorname{sl}(n+1)$ AKNS theory is defined by the linear matrix problem [1]

$$
\begin{aligned}
A \Psi & =\partial \Psi \\
B_{m} \Psi & =\partial_{t_{m}} \Psi \quad m=2,3, \ldots
\end{aligned}
$$

for

$$
A=\lambda E+A^{0} \quad \text { with } \quad E=\frac{2 \lambda_{n} \cdot H}{\alpha_{n}^{2}}
$$

where $\lambda_{n}$ and $\alpha_{n}$ are the n-th fundamental weight and simple root of $\operatorname{sl}(n+1)$ respectively, and

$$
A^{0}=\sum_{a=1}^{n}\left(q_{a} E_{\left(\alpha_{a}+\ldots+\alpha_{n}\right)}+r_{a} E_{-\left(\alpha_{a}+\ldots+\alpha_{n}\right)}\right)
$$

and where $q_{a}$ and $r_{a}$ are the fields of the model, satisfying the Generalized Non-Linear Schrodinger equations

$$
\begin{aligned}
\frac{\partial q_{a}}{\partial t} & =\partial_{x}^{2} q_{a}-2 q_{a} \sum_{b=1}^{n} q_{b} r_{b} \\
\frac{\partial r_{a}}{\partial t} & =-\partial_{x}^{2} r_{a}+2 r_{a} \sum_{b=1}^{n} q_{b} r_{b}
\end{aligned}
$$

\footnotetext{
${ }^{3}$ Throughout this paper we will use the Chevalley basis for a Lie algebra $\mathcal{G}, H_{a}, E_{\alpha}$, satisfying $\left[H_{a}, H_{b}\right]=$ $0,\left[H_{a}, E_{\alpha}\right]=K_{\alpha a} E_{\alpha}$ and $\left[E_{\alpha}, E_{\beta}\right]$ is equal to $\epsilon(\alpha, \beta) E_{\alpha+\beta}$ if $\alpha+\beta$ is a root, to $\sum_{a=1}^{n} n_{a} H_{a}$ if $\alpha+\beta=0$, and 0 otherwise. We have denoted $K_{\alpha a}=\frac{2 \alpha \cdot \alpha_{a}}{\alpha_{a}^{2}}=\sum n_{b} K_{b a}$, with $K_{a b}$ being the Cartan matrix, and a root $\alpha$ can be expanded in terms of simple roots as $\alpha=\sum n_{a} \alpha_{a}$. The quantities $\epsilon(\alpha, \beta)$ are integers (just signs for simple laced algebras) determined by use of the Jacobi identities.
} 
The model described by the eqs. (1.5) is a representative of a hierarchy which consists of an infinite set of equations involving an infinite number of times. Successive flows are related by the recursion operator $\mathcal{R}$, as explained in [2], by

$$
\begin{aligned}
& \partial_{t_{n}}\left(\begin{array}{c}
r_{i} \\
q_{l}
\end{array}\right)=\mathcal{R}_{(i, l),(j, m)} \partial_{t_{n-1}}\left(\begin{array}{c}
r_{j} \\
q_{m}
\end{array}\right)= \\
& \left(\begin{array}{cc}
\left(-\partial+r_{k} \partial^{-1} q_{k}\right) \delta_{i j}+r_{i} \partial^{-1} q_{j} & r_{i} \partial^{-1} r_{m}+r_{m} \partial^{-1} r_{i} \\
-q_{l} \partial^{-1} q_{j}-q_{j} \partial^{-1} q_{l} & \left(\partial-q_{k} \partial^{-1} r_{k}\right) \delta_{l m}-q_{l} \partial^{-1} r_{m}
\end{array}\right) \partial_{t_{n-1}}\left(\begin{array}{c}
r_{j} \\
q_{m}
\end{array}\right)
\end{aligned}
$$

A first Hamiltonian structure is introduced from a Poisson bracket

$$
P_{1}(x, y)=\left(\begin{array}{ll}
\left\{r_{i}, r_{j}\right\} & \left\{r_{i}, q_{m}\right\} \\
\left\{q_{l}, r_{j}\right\} & \left\{q_{l}, q_{m}\right\}
\end{array}\right)=\left(\begin{array}{cc}
0 & -I \\
I & 0
\end{array}\right) \delta(x-y)
$$

A second Poisson bracket structure can be obtained from the recursion operator

$$
\mathcal{R}=P_{2} P_{1}^{-1}
$$

leading to

$$
\begin{aligned}
& P_{2}(x, y)=\left(\begin{array}{cc}
0 & \delta_{i m} \\
\delta_{l j} & 0
\end{array}\right) \partial_{x} \delta(x-y)+ \\
& \left(\begin{array}{cc}
r_{i}(x) r_{j}(y)+r_{j}(x) r_{i}(y) & -\delta_{i m} \sum_{k} r_{k}(x) q_{k}(y)-r_{i}(x) q_{m}(y) \\
-\delta_{l j} \sum_{k} q_{k}(x) r_{k}(y)-q_{l}(x) r_{j}(y) & q_{l}(x) q_{m}(y)+q_{m}(x) q_{l}(y)
\end{array}\right) \partial_{x}^{-1} \delta(x-y)
\end{aligned}
$$

We say two brackets $\{\cdot, \cdot\}_{i}$ and $\{\cdot, \cdot\}_{j}$ are compatible if

$$
K_{i j}(A, B, C) \equiv J_{i j}(A, B, C)+J_{j i}(A, B, C)=0
$$

where

$$
J_{i j}(A, B, C) \equiv\left\{\{A, B\}_{i}, C\right\}_{j}+\left\{\{C, A\}_{i}, B\right\}_{j}+\left\{\{B, C\}_{i}, A\right\}_{j}
$$

As we have mentioned in the introduction the bracket $P_{2}$ does not necessarily satisfy the Jacobi identities. That can be easily seen by considering the Jacobi identity for the three fields $r_{1}(x), r_{1}(y)$ and $r_{2}(z)$. The source of the problem lies in the way one expresses the quantity $\partial_{x}^{-1} \delta(x-y)$ in terms of Heaviside's $\Theta$ functions. The generic form of such relation is

$$
\partial_{x}^{-1} \delta(x-y)=\gamma \Theta(x-y)-(1-\gamma) \Theta(y-x)
$$

since (see (3.3))

$$
\partial_{x} \partial_{x}^{-1} \delta(x-y)=\delta(x-y) \quad \text { for any } \gamma
$$




\section{I.I. The $\operatorname{sl}(2)$ case}

As a first example consider the $\operatorname{sl}(2)$ case, where the first and second brackets are given respectively by

$$
\{r(x), r(y)\}_{1}=\{q(x), q(y)\}_{1}=0 \quad\{r(x), q(y)\}_{1}=-\delta(x-y)
$$

and (from now on we will use primes to denote derivatives w.r.t. the argument of the function, e.g. $\left.\partial_{x} \delta(x-y) \equiv \delta^{\prime}(x-y)\right)$

$$
\begin{aligned}
& \{r(x), r(y)\}_{2}=2 r(x) r(y) \partial_{x}^{-1} \delta(x-y) \\
& \{q(x), q(y)\}_{2}=2 q(x) q(y) \partial_{x}^{-1} \delta(x-y) \\
& \{r(x), q(y)\}_{2}=\delta^{\prime}(x-y)-2 r(x) q(y) \partial_{x}^{-1} \delta(x-y)
\end{aligned}
$$

Since the bracket has to be antisymmetric under the exchange of its entries, we choose, on (1.15) and (1.16), the constant $\gamma$ introduced in (1.12) to be $\frac{1}{2}$. Then the Jacobi identity implies that we have to make the same value for $\gamma$ in (1.17).

We can also introduce a third bracket by the relation

$$
P_{3} \equiv P_{2} P_{1}^{-1} P_{2}
$$

giving

$$
\begin{aligned}
\{r(x), r(y)\}_{3} & =-\left(r(x) r^{\prime}(y)+r^{\prime}(x) r(y)\right) \epsilon(x-y) \\
\{q(x), q(y)\}_{3} & =\left(q(x) q^{\prime}(y)+q^{\prime}(x) q(y)\right) \epsilon(x-y) \\
\{r(x), q(y)\}_{3} & =-\delta^{\prime \prime}(x-y)-\left(r(x) q^{\prime}(y)-r^{\prime}(x) q(y)\right) \epsilon(x-y) \\
& +4 r(x) q(x) \delta(x-y)
\end{aligned}
$$

We have checked, using the Mathematica program, that the three brackets defined above satisfy the Jacobi identities, and in addition, that they are all compatible with each other, in the sense of (1.10). The above results establish that the $s l(2)$-AKNS system is triHamiltonian.

\section{I.II. The $\operatorname{sl}(3)$ case}

We have checked that in order for the Jacobi identity to be satisfied, the second bracket for the $s l(3)$-AKNS system given in (1.10), one can not choose the value of $\gamma$ introduced in (1.12), to be the same for all brackets. Because of antysymmetry, one has to take $\gamma=\frac{1}{2}$ for brackets involving the same type of fields, and for the other brackets we determine the values of $\gamma$ imposing the Jacobi identity. The result we got, using Mathematica, is that there are

two possibilities for the choices of $\gamma$, specified below by the parameter $\eta$ which can take the values \pm 1 .

$$
\begin{array}{ll}
\left\{r_{i}(x), r_{i}(y)\right\}_{2}=r_{i}(x) r_{i}(y) \epsilon(x-y) & \text { for } i=1,2 \\
\left\{q_{i}(x), q_{i}(y)\right\}_{2}=q_{i}(x) q_{i}(y) \epsilon(x-y) & \text { for } i=1,2
\end{array}
$$




$$
\begin{aligned}
\left\{r_{i}(x), q_{i}(y)\right\}_{2} & =\delta^{\prime}(x-y)-r_{i}(x) q_{i}(y) \epsilon(x-y) \\
& +\eta \sum_{j=1}^{2} \varepsilon_{i j} r_{j}(x) q_{j}(y) \Theta\left(\eta \varepsilon_{i j}(y-x)\right) \quad \text { for } i=1,2 \\
\left\{r_{1}(x), r_{2}(y)\right\}_{2} & =r_{1}(x) r_{2}(y) \Theta(x-y)-\eta r_{1}(y) r_{2}(x) \Theta(\eta(y-x)) \\
\left\{q_{1}(x), q_{2}(y)\right\}_{2} & =q_{1}(x) q_{2}(y) \Theta(x-y)+\eta q_{1}(y) q_{2}(x) \Theta(\eta(x-y)) \\
\left\{r_{1}(x), q_{2}(y)\right\}_{2} & =-r_{1}(x) q_{2}(y) \Theta(x-y) \\
\left\{r_{2}(x), q_{1}(y)\right\}_{2} & =r_{2}(x) q_{1}(y) \Theta(y-x)
\end{aligned}
$$

where $\varepsilon_{i j}$ is antisymmetric and $\varepsilon_{12}=1$.

It is now straightforward to show that the first bracket defined in (1.7) is compatible with the above second brackets for the two choices of $\eta$, namely $\eta= \pm 1$. Therefore the $s l(3)$-AKNS system is bi-Hamiltonian. Recently, Liu [3] has argued that the $s l(3)$-AKNS system is bi-Hamiltonian. However, it was not taken into account the fact that the Jacobi identity may not be satisfied, if a careful prescription for $\partial_{x}^{-1} \delta(x-y)$ (see (1.12)) is not made.

Let us mention that through (1.18) we can calculate the third bracket for this case. However, we need to check the Jacobi identity and the compatibility condition (1.10) with the other two brackets in order to establish that the system is indeed tri-Hamiltonian.

\section{DNLS}

The Generalized $s l(n+1)$-DNLS system is defined by (1.1)-(1.2) for $A$ given by [四]

$$
A=\lambda^{2} E+\lambda A^{0}
$$

with $E$ given by (1.3), and

$$
A^{0}=\sum_{a=1}^{n}\left(-q_{a} E_{\left(\alpha_{a}+\ldots+\alpha_{n}\right)}+r_{a} E_{-\left(\alpha_{a}+\ldots+\alpha_{n}\right)}\right)
$$

The corresponding Generalized DNLS equations of motion are

$$
\begin{aligned}
& \frac{\partial q_{a}}{\partial t}=\partial_{x}^{2} q_{a}+2 \partial_{x}\left(q_{a} \sum_{b=1}^{n} q_{b} r_{b}\right) \\
& \frac{\partial r_{a}}{\partial t}=-\partial_{x}^{2} r_{a}+2 \partial_{x}\left(r_{a} \sum_{b=1}^{n} q_{b} r_{b}\right)
\end{aligned}
$$

Let us now discuss the recursion operator for such system. Consider the Zakharov-Shabat equations for consecutive times $t_{m}$ and $t_{m-1}$

$$
\begin{aligned}
\partial_{t_{m}} A-\partial_{x} B_{m}+\left[A, B_{m}\right] & =0 \\
\partial_{t_{m-1}} A-\partial_{x} B_{m-1}+\left[A, B_{m-1}\right] & =0
\end{aligned}
$$

In order to determine the general solution of these equations we take the ansatz

$$
B_{m}=\lambda^{2} B_{m-1}+\lambda^{2} C_{m}+\lambda D_{m}+Y_{m}
$$


Multiplying (2.4) by $\lambda$, (2.5) by $\lambda^{3}$, taking the difference and using the ansatz (2.6), one gets

$$
\begin{aligned}
\lambda \partial_{t_{m}} A^{0} & -\lambda^{3} \partial_{t_{m-1}} A^{0}-\partial_{x}\left(\lambda^{2} C_{m}+\lambda D_{m}+Y_{m}\right)-\lambda^{2}\left[E, \lambda^{2} C_{m}+\lambda D_{m}+Y_{m}\right] \\
& +\lambda\left[A^{0}, \lambda^{2} C_{m}+\lambda D_{m}+Y_{m}\right]=0
\end{aligned}
$$

The $\lambda$ independent components yields

$$
\partial_{x} Y_{m}=0
$$

so that we can choose $Y_{m}=0$. The other components yields the following equations

$$
\begin{aligned}
\partial_{t_{m}} A^{0}-\partial_{x} D_{m} & =0 \\
\partial_{x} C_{m}-\left[A^{0}, D_{m}\right] & =0 \\
\partial_{t_{m-1}} A^{0}-\left[E, D_{m}\right]-\left[A^{0}, C_{m}\right] & =0 \\
{\left[E, C_{m}\right] } & =0
\end{aligned}
$$

From the last equation we conclude that $C_{m} \in \operatorname{Ker}(\operatorname{ad} E)$. Since $A^{0} \in \operatorname{Im}(\operatorname{ad} E)$, the first equation implies that also $D_{m} \in \operatorname{Im}(\operatorname{ad} E)$ (except for a $x$ independent component in Ker $(\operatorname{ad} E)$ which we do not consider).

\section{II.I. The $\operatorname{sl}(2)$ case}

In this case one hasf

$$
E=\frac{1}{2} \sigma_{3}, \quad A^{0}=-q \sigma_{+}+r \sigma_{-}
$$

and so, since $C_{m} \in \operatorname{Ker}(\operatorname{ad} E)$ and $D_{m} \in \operatorname{Im}(\operatorname{ad} E)$ one has

$$
C_{m}=c_{m} \sigma_{3}, \quad D_{m}=d_{m}^{+} \sigma_{+}+d_{m}^{-} \sigma_{-}
$$

Replacing (2.13) into (2.10) and (2.11) one gets

$$
\begin{aligned}
\partial_{x} c_{m}+q d_{m}^{-}+r d_{m}^{+} & =0 \\
\partial_{t_{m-1}} q-d_{m}^{+}-2 q c_{m} & =0 \\
\partial_{t_{m-1}} r+d_{m}^{-}-2 r c_{m} & =0
\end{aligned}
$$

From these equations one finds

$$
c_{m}=\partial_{x}^{-1} \partial_{t_{m-1}}(r q)
$$

Replacing into (2.16) and (2.17) leads to

$$
\begin{aligned}
& d_{m}^{+}=-\partial_{t_{m-1}} q-2 q \partial_{x}^{-1} \partial_{t_{m-1}}(r q) \\
& d_{m}^{-}=-\partial_{t_{m-1}} r+2 r \partial_{x}^{-1} \partial_{t_{m-1}}(r q)
\end{aligned}
$$

\footnotetext{
${ }^{4}$ Here $\sigma_{3, \pm}$ stands for the combinations of the Pauli matrices such that, $\left[\sigma_{3}, \sigma_{ \pm}\right]= \pm 2 \sigma_{ \pm}$and $\left[\sigma_{+}, \sigma_{-}\right]=$ $\sigma_{3}$.
} 
Substituting into (2.9), one can write it in the form of (1.6) with the recursion operator given by

$$
\begin{aligned}
R(x, y) & =\left(\begin{array}{cc}
-1 & 0 \\
0 & 1
\end{array}\right) \partial_{x} \delta(x-y)+2\left(\begin{array}{cc}
q(x) r(x) & r(x)^{2} \\
q(x)^{2} & q(x) r(x)
\end{array}\right) \delta(x-y) \\
& +2\left(\begin{array}{cc}
r^{\prime}(x) q(y) & r^{\prime}(x) r(y) \\
q^{\prime}(x) q(y) & q^{\prime}(x) r(y)
\end{array}\right) \partial_{x}^{-1} \delta(x-y)
\end{aligned}
$$

One can verifies that the recursion operator can be decomposed into

$$
R=P_{3} P_{2}^{-1}
$$

where

$$
P_{3}(x, y)=\left(\begin{array}{rr}
0 & \delta^{\prime}(x-y) \\
\delta^{\prime}(x-y) & 0
\end{array}\right)
$$

and

$$
P_{2}(x, y)=\left(\begin{array}{cc}
0 & -1 \\
1 & 0
\end{array}\right) \delta(x-y)+2\left(\begin{array}{cc}
r(x) r(y) & -r(x) q(y) \\
-q(x) r(y) & q(x) q(y)
\end{array}\right) \partial_{x}^{-1} \delta(x-y)
$$

The brackets associated to the above $P_{2}$ and $P_{3}$ were proposed in [6], by choosing the prescription $\gamma=\frac{1}{2}$ in (1.12), i.e.

$$
2 \partial_{x}^{-1} \delta(x-y)=\epsilon(x-y)
$$

in all entries of (2.24).

We can obtain a further bracket, denoted $P_{4}$, by

$$
R=P_{4} P_{3}^{-1}
$$

It gives

$$
\begin{aligned}
\{r(x), r(y)\}_{4} & =-r^{\prime}(x) r^{\prime}(y) \epsilon(x-y)+2 r^{\prime}(x) r(y) \delta(x-y)+2 r(x)^{2} \delta^{\prime}(x-y)(2 . \\
\{q(x), q(y)\}_{4} & =-q^{\prime}(x) q^{\prime}(y) \epsilon(x-y)+2 q^{\prime}(x) q(y) \delta(x-y)+2 q(x)^{2} \delta^{\prime}(x-y)(2 \\
\{r(x), q(y)\}_{4} & =-\delta^{\prime \prime}(x-y)-r^{\prime}(x) q^{\prime}(y) \epsilon(x-y)+2 r^{\prime}(x) q(x) \delta(x-y) \\
& +2 r(x) q(x) \delta^{\prime}(x-y)
\end{aligned}
$$

The third and fourth brackets, $P_{3}$ and $P_{4}$, were introduced in ref. [7], from which they constructed the recursion operator $R$ through (2.26).

We have verified, using the Mathematica program, that the three brackets $P_{2}, P_{3}$ and $P_{4}$ satify the Jacobi identities and are compatible with each other in the sense of (1.10). Therefore, we have established that such system is tri-Hamiltonian. 


\section{II.II. The $\operatorname{sl}(3)$ case}

Let us parametrize, in this case, the quantities introduced in (2.6) as

$$
\begin{aligned}
C_{m} & =c_{m}^{\left(\alpha_{1}\right)} E_{\alpha_{1}}+c_{m}^{\left(-\alpha_{1}\right)} E_{-\alpha_{1}}+c_{m}^{(1)} H_{1}+c_{m}^{(2)} H_{2} \\
D_{m} & =d_{m}^{\left(\alpha_{1}+\alpha_{2}\right)} E_{\alpha_{1}+\alpha_{2}}+d_{m}^{\left(\alpha_{2}\right)} E_{\alpha_{2}}+d_{m}^{\left(-\alpha_{1}-\alpha_{2}\right)} E_{-\alpha_{1}-\alpha_{2}}+d_{m}^{\left(-\alpha_{2}\right)} E_{-\alpha_{2}} \\
A^{0} & =-q_{1} E_{\alpha_{1}+\alpha_{2}}-q_{2} E_{\alpha_{2}}+r_{1} E_{-\alpha_{1}-\alpha_{2}}+r_{2} E_{-\alpha_{2}}
\end{aligned}
$$

Substituting in (2.10) one gets

$$
\begin{aligned}
\partial_{x} c_{m}^{\left(\alpha_{1}\right)} & =-q_{1} d_{m}^{\left(-\alpha_{2}\right)}-r_{2} d_{m}^{\left(\alpha_{1}+\alpha_{2}\right)} \\
\partial_{x} c_{m}^{\left(-\alpha_{1}\right)} & =-q_{2} d_{m}^{\left(-\alpha_{1}-\alpha_{2}\right)}-r_{1} d_{m}^{\left(\alpha_{2}\right)} \\
\partial_{x} c_{m}^{(1)} & =-q_{1} d_{m}^{\left(-\alpha_{1}-\alpha_{2}\right)}-r_{1} d_{m}^{\left(\alpha_{1}+\alpha_{2}\right)} \\
\partial_{x} c_{m}^{(2)} & =-q_{1} d_{m}^{\left(-\alpha_{1}-\alpha_{2}\right)}-r_{1} d_{m}^{\left(\left(_{1}+\alpha_{2}\right)\right.}-q_{2} d_{m}^{\left(-\alpha_{2}\right)}-r_{2} d_{m}^{\left(\alpha_{2}\right)}
\end{aligned}
$$

whilst (2.11) leads to

$$
\begin{aligned}
d_{m}^{\left(\alpha_{1}+\alpha_{2}\right)} & =-\partial_{t_{m-1}} q_{1}-q_{1}\left(c_{m}^{(1)}+c_{m}^{(2)}\right)-q_{2} c_{m}^{\left(\alpha_{1}\right)} \\
d_{m}^{\left(\alpha_{2}\right)} & =-\partial_{t_{m-1}} q_{2}-q_{2}\left(-c_{m}^{(1)}+2 c_{m}^{(2)}\right)-q_{1} c_{m}^{\left(-\alpha_{1}\right)} \\
d_{m}^{\left(-\alpha_{1}-\alpha_{2}\right)} & =-\partial_{t_{m-1}} r_{1}+r_{1}\left(c_{m}^{(1)}+c_{m}^{(2)}\right)+r_{2} c_{m}^{\left(-\alpha_{1}\right)} \\
d_{m}^{\left(-\alpha_{2}\right)} & =-\partial_{t_{m-1}} r_{2}+r_{2}\left(-c_{m}^{(1)}+2 c_{m}^{(2)}\right)+r_{1} c_{m}^{\left(\alpha_{1}\right)}
\end{aligned}
$$

From the eqs. (2.33)-(2.40) one gets

$$
c_{m}^{(2)}=\partial_{x}^{-1} \partial_{t_{m-1}}\left(r_{1} q_{1}+r_{2} q_{2}\right)
$$

and

$$
\begin{aligned}
\partial_{x} c_{m}^{\left(\alpha_{1}\right)}-q_{1} r_{2}\left(2 c_{m}^{(1)}-c_{m}^{(2)}\right)-\left(r_{2} q_{2}-r_{1} q_{1}\right) c_{m}^{\left(\alpha_{1}\right)} & =\partial_{t_{m-1}}\left(q_{1} r_{2}\right) \\
\partial_{x} c_{m}^{\left(-\alpha_{1}\right)}+q_{2} r_{1}\left(2 c_{m}^{(1)}-c_{m}^{(2)}\right)+\left(r_{2} q_{2}-r_{1} q_{1}\right) c_{m}^{\left(-\alpha_{1}\right)} & =\partial_{t_{m-1}}\left(q_{2} r_{1}\right) \\
\partial_{x} c_{m}^{(1)}-r_{1} q_{2} c_{m}^{\left(\alpha_{1}\right)}+r_{2} q_{1} c_{m}^{\left(-\alpha_{1}\right)} & =\partial_{t_{m-1}}\left(q_{1} r_{1}\right)
\end{aligned}
$$

One can write the above system of differential equations in a more elegant way, introducing

$$
c_{m}^{ \pm} \equiv c_{m}^{\alpha_{1}} \pm c_{m}^{-\alpha_{1}}, \quad c_{m}^{0} \equiv 2 c_{m}^{1}-c_{m}^{2}
$$

and also

$$
\gamma_{ \pm} \equiv q_{1} r_{2} \pm q_{2} r_{1}, \quad \gamma_{0} \equiv q_{1} r 1-q_{2} r_{2}
$$

Now, introduce the matrices

$$
c \equiv\left(\begin{array}{c}
c_{+} \\
c_{0} \\
c_{-}
\end{array}\right) ; \quad \gamma \equiv\left(\begin{array}{c}
\gamma_{+} \\
\gamma_{0} \\
\gamma_{-}
\end{array}\right)
$$


and

$$
W \equiv\left(\begin{array}{ccc}
0 & -\gamma_{-} & \gamma_{0} \\
\gamma_{-} & 0 & -\gamma_{+} \\
\gamma_{0} & -\gamma_{+} & 0
\end{array}\right)
$$

Then, the solution for the system (2.42)-(2.44) can be written formally as

$$
c=D_{x}^{-1} \partial_{t_{m-1}} \gamma \quad D_{x} \equiv \partial_{x}+W
$$

Notice that $W$ is a matrix in the adjoint of $s l(2)$, and therefore in the actual integration of the above equations such algebraic structure should play an important role.

\section{Appendix}

We give here some definitions and relations involving delta and Heaviside functions.

The Heaviside function is defined by

$$
\Theta(x-y) \equiv \begin{cases}1 & \text { for } x>y \\ 0 & \text { for } x<y \\ \frac{1}{2} & \text { for } x=y\end{cases}
$$

We also introduce the sign function as

$$
\epsilon(x-y) \equiv \Theta(x-y)-\Theta(y-x)
$$

One also has

$$
\partial_{x} \Theta(x-y)=-\partial_{y} \Theta(x-y)=\delta(x-y)
$$

and so

$$
\partial_{x} \epsilon(x-y)=-\partial_{y} \epsilon(x-y)=2 \delta(x-y)
$$

In order to verifiy Jacobi identities and other relations involving fields at different points we used the strategy of using delta functions and its derivatives to try to write the fields at the same point. The relations used can be derived from the identity

$$
f(y) \delta(x-y)=f(x) \delta(x-y)
$$

Indeed, differentiating it one gets

$$
f(y) \frac{d^{n} \delta(x-y)}{d x^{n}}=\sum_{l=0}^{n}\left(\begin{array}{c}
n \\
l
\end{array}\right) \frac{d^{n-l} \delta(x-y)}{d x^{n-l}} \frac{d^{l} f(x)}{d x^{l}}
$$

We also have used the identities

$$
\begin{aligned}
& \Theta(y-x) \Theta(z-x)-\Theta(y-z) \Theta(z-x)-\Theta(y-x) \Theta(z-y)=0 \\
& \Theta(x-y) \Theta(z-x)+\Theta(y-x) \Theta(z-y)-\Theta(z-x) \Theta(z-y)=0
\end{aligned}
$$

\section{Acknowledgements}

We are greatful to H. Aratyn for the correspondence on the subject. 


\section{References}

[1] A.P. Fordy and P.P. Kulish, Commun. Math. Phys. 89 (1983) 427; A.P. Fordy, in Soliton Theory: a Survey of Results, (ed. A.P. Fordy) University Press, Manchester (1990), pg. 315

[2] H. Aratyn, J.F. Gomes and A.H. Zimerman, J. Math. Phys. 36 (1995) 3419-3442, hepth/9408104.

[3] Q.P. Liu; J. Math. Phys. 37 (1996) 2307-2314.

[4] A.P. Fordy; J. Physics A17 (1984) 1235-1245.

[5] F. Magri; J. Math. Phys. 19 (1978) 1156.

[6] H. Aratyn, L.A. Ferreira, J.F. Gomes and A.H. Zimerman, On $W_{\infty}$ algebras, gauge equivalence of KP hierarchies, two-boson realizations and their KdV reductions, Proceedings of the VII J.A. Swieca Summer School, section: Particles and Fields, January/93, eds. O.J.P. Éboli and V.O. Rivelles (World Scientific, 1994), pages 630-666, (see eqs. (6.43)and (6.47)), hep-th/9304152.

[7] R. Willox, W. Hereman and F. Verheest; Physica Scripta 52 (1995) 21. 